\title{
Single-Macromolecular Level Imaging of a Hydrogel Structure
}

\author{
Ryuji Kiyama, Takayuki Nonoyama, Tomas Sedlacik, Hiroshi Jinnai, and Jian Ping Gong*
}

R. Kiyama, Graduate School of Life Science, Hokkaido University, Sapporo 001-0021, Japan

Prof. T. Nonoyama, Dr. T. Sedlacik, Prof. J. P. Gong, Faculty of Advanced Life Science, Hokkaido University, Sapporo 001-0021, Japan

Prof. T. Nonoyama, Dr. T. Sedlacik, Prof. J. P. Gong, Global Station for Soft Matter, Global Institution for Collaborative Research and Education (GI-CoRE), Hokkaido University, Sapporo 001-0021, Japan

Prof. H Jinnai, Institute of Multidisciplinary Research for Advanced Materials, Tohoku University, Sendai 980-8577, Japan

Prof. J. P. Gong, Institute for Chemical Reaction Design and Discovery (WPI-ICReDD), Hokkaido University, Sapporo, 001-0021, Japan

Tel: +81-11-706-9011; E-mail: gong@ sci.hokudai.ac.jp

Keywords: Hydrogel, Direct observation, Transmission electron microscope, Double Network gel, Mineral staining, Inhomogeneity, Dangling chain

Hydrogels are promising materials for several applications, including cell scaffolds and artificial load-bearing substitutes (cartilages, ligaments, tendons, etc.). Direct observation of the nanoscale polymer network of hydrogels is essential in understanding its properties.

However, imaging of individual network strands at the molecular level is not achieved yet due to the lack of suitable methods. Herein, for the first time, we developed a novel mineralstaining method and network fixation method for transmission electron microscopy observation to visualize the hydrogel network in its unperturbed conformation with nanometer 
resolution. Surface network observation indicates that the length of surface dangling chains, which play a major role in friction and wetting, can be estimated from the gel mesh size.

Moreover, bulk observations reveals a hierarchical formation mechanism of gel heterogeneity.

These observations have the great potential to advance gel science by providing comprehensive perspective that link bulk gel properties with nanoscale.

\section{Introduction}

Hydrogel is a water-abundant three-dimensional polymer network that is a promising soft material for various applications, including bioengineering, soft robotics, and stretchable electronics, owing to its high water content, low friction, permeability of small molecules and ions, flexibility, and biocompatibility ${ }^{[1-4]}$. The surface and bulk properties of hydrogels strongly depend on their molecular structure. However, state-of-the-art approaches to characterise the hydrogel network structure, especially synthetic hydrogel, are still limited at the average structure level derived from bulk measurements, such as the mesh size of a network is determined from the bulk modulus or X-ray/light scattering ${ }^{[5-7]}$. Direct observation of individual network strands at the molecular level is yet to be achieved due to insufficient suitable methods. Transmission electron microscopy (TEM) and atomic force microscopy (AFM) are the most commonly used imaging techniques to directly observe the nanostructures of materials. Compared to AFM, TEM is more suitable for gels as 
it can eliminate the influence of the thermal motion of the network using resin substitution or water freezing at cryogenic temperatures ${ }^{[8,9]}$. However, existing TEM observation methods, such as electron staining, phase contrast, and scanning $\mathrm{TEM}^{[10-13]}$, cannot produce a sufficient contrast for chemical gels consisting of thin polymer chains; therefore, a contrast enhancement method is desired. We considered the development of an improved staining method to be the most promising approach in terms of simplicity and contrast enhancement efficiency. structure of synthetic polyelectrolyte hydrogels in the unperturbed state with approximately $10 \mathrm{~nm}$ resolution. We introduced a skeleton neutral polymer network in the polyelectrolyte hydrogel to fix the conformation of the polyelectrolyte strands during specimen preparation. We adopted the mineralization of amorphous ferric oxide (AFO) nanoparticles to stain the polyelectrolyte strands in the unperturbed state. This method allowed the imaging of a network structure in a wide mesh size range (tens to hundreds of nanometers) for the first time and surface molecular structure of hydrogels. These observations provide important insights for the friction and fracture of hydrogels.

\section{Results and Discussion}

\subsection{Mineral nanoparticle staining and double-network fixation method}


double bond with one heavy atom does not provide enough contrast to the sparse and extremely thin network of hydrogels. Thus, we used inorganic nanoparticles as a bigger marker.

To accurately attach the nanoparticles to hydrogel network, we used the heterogeneous nucleation of ferric oxide ${ }^{[14]}$. Poly(2-acrylamido-2-methyl propanesulfonic acid) (PAMPS) hydrogel was adopted since sulfonic acid functional groups are expected to function as nucleation points for the growth of ferric oxide particles. PAMPS gel was synthesized by radical Figzation, resulting in its significant swelling in water due to its polyelectrolyte nature, thereby reaching a polymer weight fraction as low as $0.3-5 \mathrm{wt} . \%$. The heterogeneous nucleation of amorphous ferric oxide was gently performed in the gel by iron (III) hydrolysis and nano-sized AFO particles grew along the polymer network (Figure 1a). However, this AFO deposition caused significant irreversible shrinking of the PAMPS gel due to the high ionic osmotic pressure of the stain solution and intermolecular cross-linking (Figure 1b and Figure S1). Therefore, direct AFO deposition on a single PAMPS gel network can cause the collapse and aggregation of the polymer strands in the gel. 
a

\section{Polyelectrolyte gel}

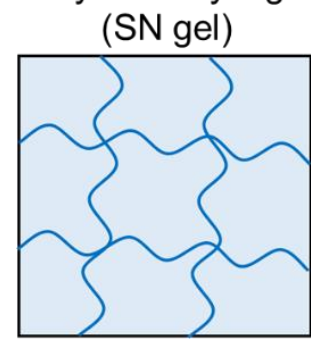

With skeleton network (DN gel)

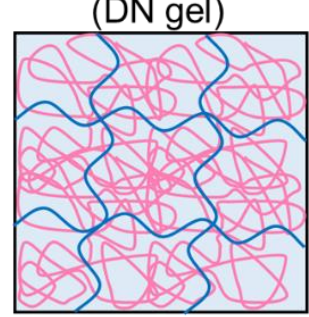

\section{Mineral staining}

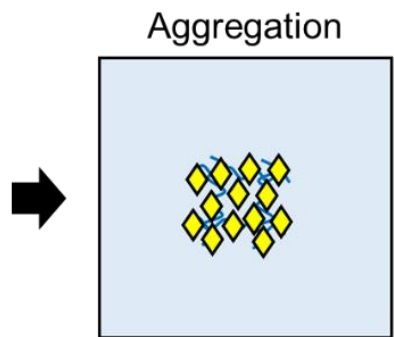

Structure preserved

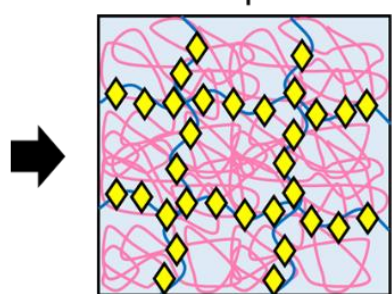

$$
\begin{array}{ll}
\curvearrowright & : \text { Polyelectrolyte } \\
\diamond & : \text { : Neutral polymer } \\
& : \text { Mineral }
\end{array}
$$

b

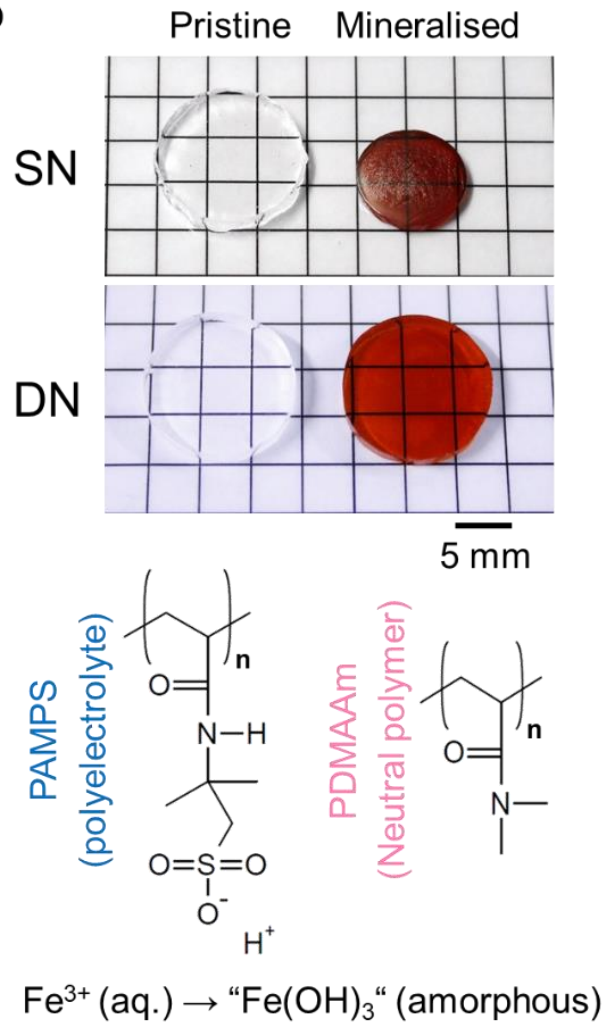

Figure 1. (a) Schematic illustration of the double-network method to stain a polyelectrolyte hydrogel without causing a collapse of the polymer network strands. (b) Appearance of the PAMPS-4 single network gel (SN) and double-network gel (DN) with poly(dimethylacrylamide) (PDMAAm) before and after mineral staining (upper), and chemical structure of PAMPS and PDMAAm, and the mineralization reaction during staining (lower).

To prevent network structure changes during staining, we applied the double-network (DN) method. DN method was originally developed to toughen the brittle polyelectrolyte gels by inducing excess amount of neutral polymer network into the polyelectrolyte ge ${ }^{[15-17]}$. In this study, we applied this method to fix the target structure of the polyelectrolyte hydrogel. 
As the skeleton neutral polymer network, poly(dimethylacrylamide) (PDMAAm), which is non-reactive to the staining solution, was polymerized in excess in the PAMPS hydrogel prior to AFO staining. By creating this interpenetrating DN structure with strong topological entanglement, the shrinking of the PAMPS network during staining is suppressed, as demonstrated by the small change in its gel size (Figure 1a and 1b). This is attributed to the neutral PDMAAm network that generates a large osmotic pressure to maintain gel swelling ${ }^{[18]}$. DN method is applicable to any polymer network. For this study, PDMAAm can maintain approximately the same volume of the specimen even in the resin solution for substitution. Figure S1 shows the detailed change in thickness of the PAMPS gels in each experimental step. The final thickness change is less than $10 \%$ for all PAMPS gel samples prepared with different formulations, indicating that the strands of the PAMPS gel was nearly unperturbed in the TEM specimen.

\subsection{Nanoscale TEM observation of the hydrogel network}

To confirm that AFO selectively mineralized only the PAMPS network, the PAMPS micro-gels embedded in the PDMAAm gel matrix were stained ${ }^{[19]}$. Figure $\mathbf{S 2}$ shows the optical microscopic image of the PAMPS micro-gels, TEM images of PAMPS micro-gels in the PDMAAm gel, and their corresponding schematic illustrations. The PAMPS micro-gels before mineralization (not shown in the figure) are unseen by TEM, while spherical micro- 
gels with several micrometer diameters are clearly identified after mineralization. This

confirms that AFO selectively stained the PAMPS network, but not the PDMAAm network

since the sulfonic group of PAMPS can catalyse the AFO deposition.

PAMPS/PDMAAm hydrogel comprising an interconnected porous structure with thin gel

a

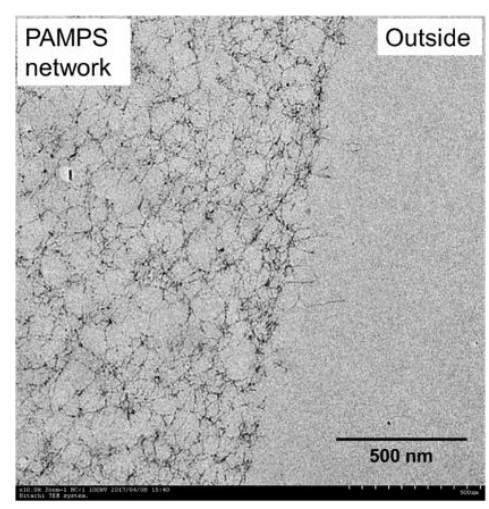

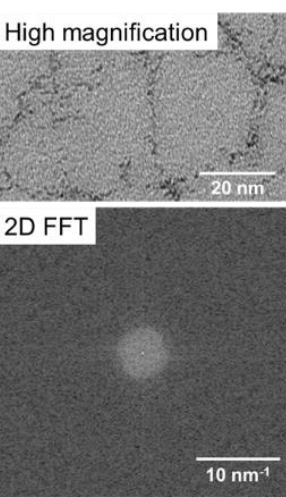
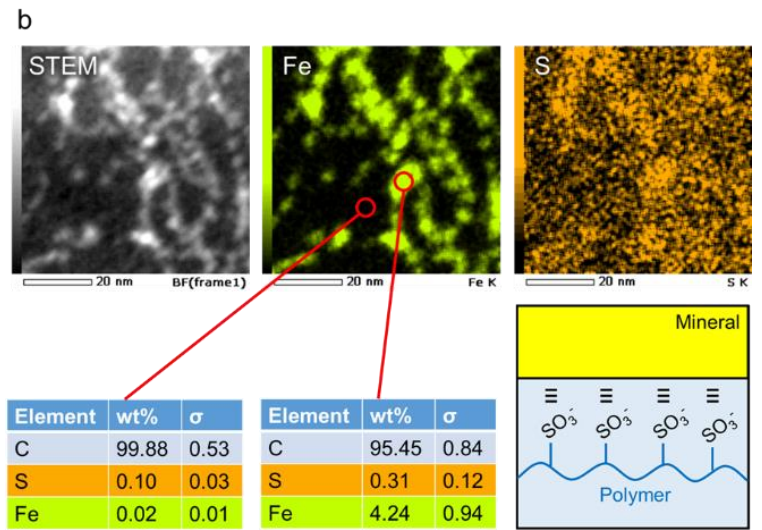

Figure 2. (a) Low and high magnification TEM images of the PAMPS gel and 2D FFT from a 
A supermacroporous PAMPS gel with a wall thickness of approximately $10 \mu \mathrm{m}$ was used for the observation.

The two-dimensional Fourier transform image of a single nanoparticle has no obvious peaks (lower right in Figure 2a), indicating its amorphous nature. Furthermore, 3D TEM was performed to obtain a 3D network structure (Supplementary Video) $)^{[21]}$, clearly presenting the $3 \mathrm{D}$ connections of the network. Notably, this is the first 3D nanoscale direct observation of a hydrogel network structure. To prove that only the PAMPS network was mineral stained at the nanoscale, element mapping (STEM-EDS) was carried out (Figure 2b). The positions of sulphur of PAMPS and iron of AFO overlap with each other on the nanoscale, confirming that only the PAMPS network was selectively stained with ferric oxide.

The high magnification TEM observation of the bulk non-porous PAMPS/PDMAAm gel is shown in Figure S3. A fine PAMPS network morphology is observed with the mesh sized in tens of nanometers, which roughly agrees with the previously reported average mesh size from small-angle X-ray scattering and mechanical estimation ${ }^{[22]}$. Considering the polymer mesh size and thickness of the specimen slice $(100 \mathrm{~nm})$, the discontinuity of the network can be attributed to the mesh structure that is frequently larger than thickness of the specimen. The accumulative strand length in the volume of the measured view $\left(1200 \times 1200 \times 100 \mathrm{~nm}^{3}\right)$ is $9100 \pm 2100 \mathrm{~nm}$, which is approximately $1 / 10$ of the contour length of $1.3 \times 10^{5} \mathrm{~nm}$ calculated from the total amount of PAMPS in the viewing volume (Figure 
151 S3a). Even considering that TEM measured a 2D projected length of approximately 0.64 the true length, there is still a large difference from the calculated value. These indicate that the observed accumulative strand length does not represent the full contour length of the polymer network and the fine structures of the polymer strands, such as blobs, were not included. Moreover, the dangling chains that should be in a collapsed conformation (Figure S3b) are

156 also not included in the observed accumulative strand length. On the other hand, this measurement provides a rough answer to the big question in the gel field, what percentage of 158 polymer chains are effective network (not dangling chains). For example, in this gel, at least one-sixth or more of the polymer chains (which probably be more than one-third because the real chains are loose and not fully extended) function as effective network. The TEM images of the PAMPS hydrogel prepared with different crosslinker densities are shown in Figure $\mathbf{S 4}$. The polymer density increases with increasing crosslinker density. In addition, the structure becomes finer, while the network morphology is unclear owing to the insufficient amount of mineral precursor to stain all polymer strands. These demonstrate that we achieved the direct observation of an actual hydrogel polymer-network structure for the first time. This method was then applied to characterise the surface and bulk structure of hydrogels.

\subsection{Surface structure of hydrogel}


molecules and ions, is an important characteristic of hydrogel materials. In nature, hyaline

171 nutrient diffusion $^{[23,24]}$. The TEM observation of the specimen prepared by our method for the

173 first time revealed the precise structure of the polymer strands at the hydrogel surface. Figure

3A(i) shows the outermost as-prepared surface of a PAMPS hydrogel synthesized on a flat glass mold. PDMAAm was induced by second polymerization with the method described in

cartilage exhibits both incredibly low friction property and semipermeability that allows the previous section. Several hundred nanometer-sized dangling chains (indicated by the black arrows) in the fully elongated state from the bulk region are observed. These surface dangling chains do not collapse as they are covalently connected to the PDMAAm network and are stretched by its swelling (Figure 3a(i)). As illustrated in Figure 3b, the PAMPS network usually has a few unreacted vinyl groups after PAMPS polymerization ${ }^{[25]}$. During the polymerization of the second skeleton network, some dangling chains with remaining vinyl groups on the PAMPS gel surface are incorporated into the PDMAAm network. Therefore, the surface of the formed PAMPS/PDMAAm DN hydrogel is covered by the second PDMAAm network partially connected with the PAMPS dangling chains ${ }^{[26]}$. The immersion of the PAMPS/PDMAAm gel in water induces the swelling of the PDMAAm network surface layer and stretching of the connected PAMPS strands. In Figure 3a(i), the approximate length of the dangling chains (several hundred nanometers) is approximately equal as that of the network mesh size in the bulk region. Therefore, these dangling strands can be assumed to be 
strands that could not find a cross-linking partner at one end. In addition, this result indicates

that the length of the surface dangling chain of the chemically crosslinked gel is comparable

to the bulk network mesh size; thus, the length of the dangling chains of the gel surface can be

chains that are covalently connected to the second network are observed as elongated strands.

As most dangling chains are not covalently connected to the second network, they collapsed dangling chains inside the gel (Figure S3b).

a
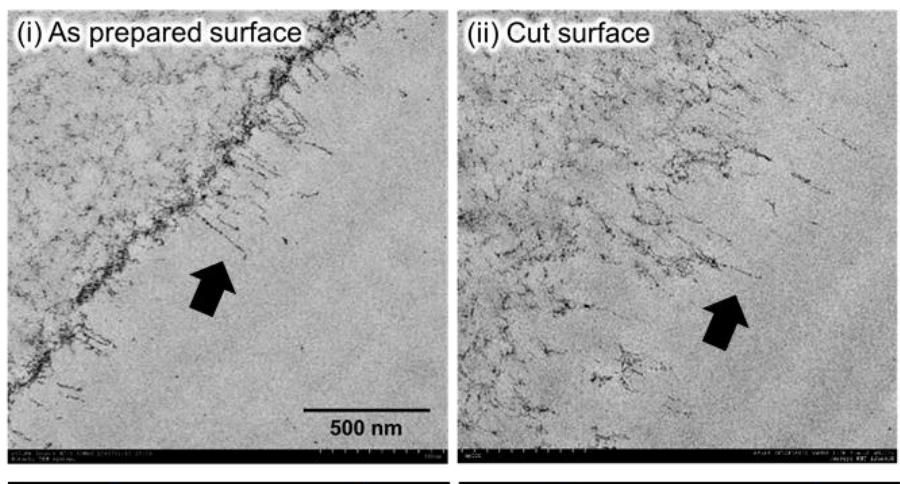

$\approx \quad$ : Residual crosslinker

- : Neutral monomer

$\sim$ : Polyelectrolyte

$\frown$ : Neutral polymer

$\diamond \quad:$ Mineral

b
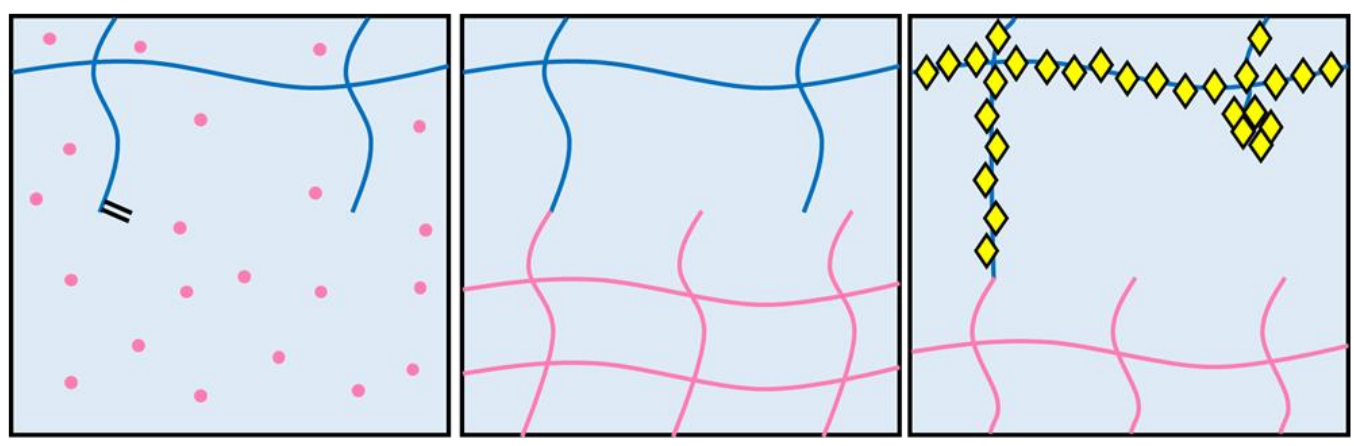

Figure 3. Surface network structure of the PAMPS-4 gel. (a) (i) As-prepared gel surface 
extension by the skeleton network. The PDMAAm network in the PAMPS gel was omitted

for schematic visibility.

We also observed the surface of the PAMPS gel cut using a fine microtome knife with

an edge thickness of $76 \mu \mathrm{m}$. The cut surface is significantly rougher than the as-prepared

surface (Figure 3a(ii)). In addition, it has a disordered network structure approximately $1 \mu \mathrm{m}$

deep from the outermost surface, while the mesh structure is maintained in the inner region.

The length of the created dangling chains at the surface is nearly equal to the bulk mesh size

since these dangling chains are originated from the mesh. Considering that cutting creates two

fracture surfaces, the damage zone has a total depth of approximately $2 \mu \mathrm{m}$, suggesting that

brittle hydrogel is fractured near the surface, despite the cutting edge thickness of $76 \mu \mathrm{m}$. This macroscopic fracture.

\subsection{Heterogeneity of the hydrogel}

217 fracture of the material as it is governed by local structure, not by the average structure ${ }^{[27,28]}$.

218 Particularly, fracture is always originated in the largest defect in a material. Here, we applied

219 the developed method to directly observe the defects in hydrogels.

Figure 4 shows the TEM images of a bulk PAMPS hydrogel and its schematic 
illustrations. The PAMPS hydrogel contains several local voids that are approximately $10 \mu \mathrm{m}$

222 (Figure 4a-c). These voids do not have a PAMPS network. The origin of defects is the

223 nonhomogeneous formation of the micro-gels ${ }^{[29,30]}$. In the initial stage of hydrogel

224 polymerization from the monomer and crosslinker, several micro-gels are instantaneously

225 formed with non-uniform spacing. When these microgels grow and coalesce together to form

226 the bulk gel, a void structure is formed where there are no micro-gels. These micro-scale

227 defects are not observed in small particle gels with a diameter of several micrometers (Figure

228 S2). Hence, a small gel is relatively homogeneous and free of large defects, while defect

229 formation is inevitable for a bulk gel, thereby affecting its mechanical properties. This

230 observation allows a simultaneous observation of the hydrogel structure at multiple scales.

231 Figure 4d-f shows a high magnification image of a void and its schematic illustrations.

232 Polymer strands around the void are oriented along the defect circumference, indicating that

233 these strands are highly elongated than those farther from the voids. This result denotes that

234 microscale heterogeneity, such as voids, induces nanoscale heterogeneity. 

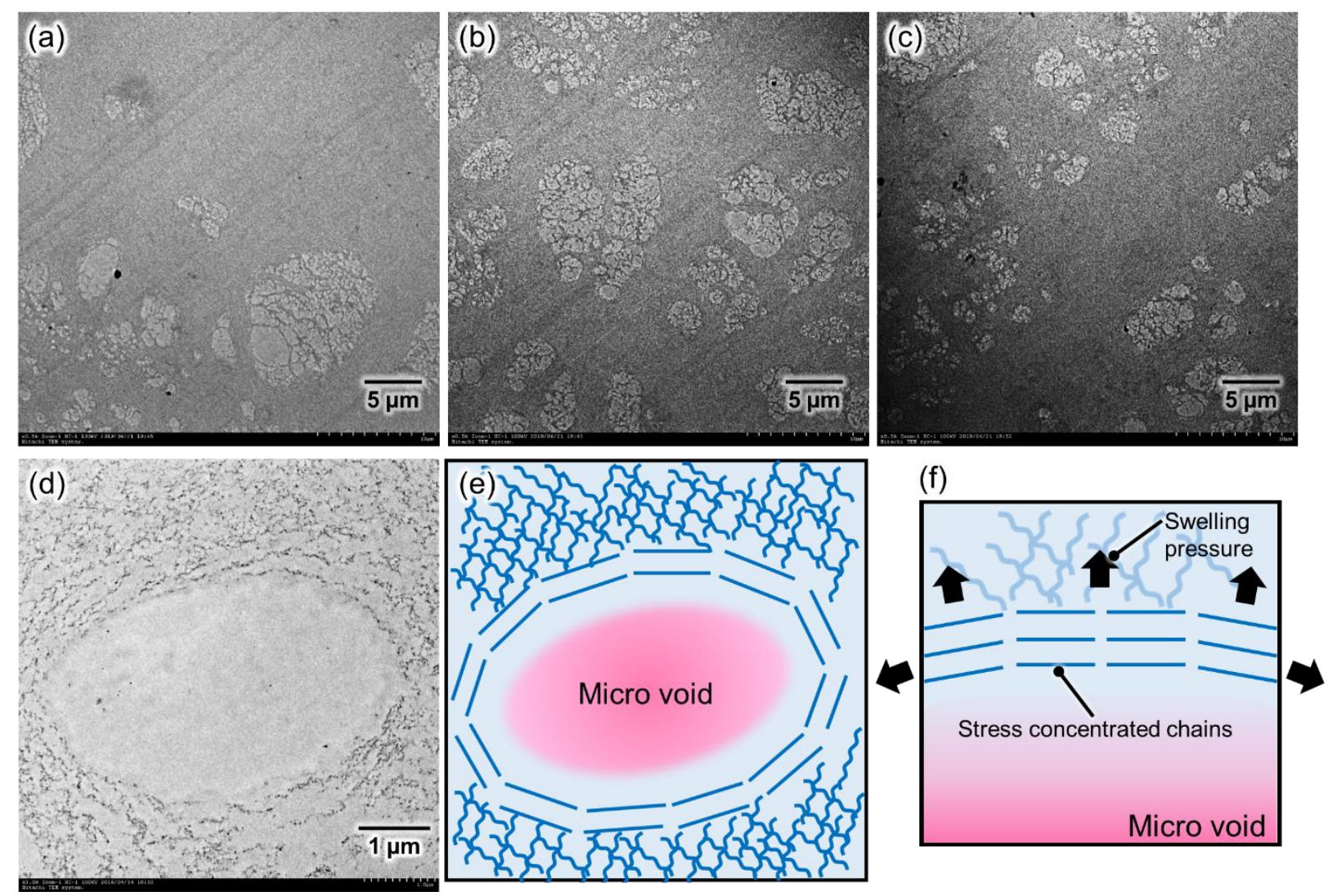

Figure 4. Micro-scale network defects in the bulk PAMPS-4 gel. (a)-(c) Low magnification

TEM images at different locations. The white areas are microvoids without a PAMPS

network. (d)-(f) High magnification TEM image around a microvoid and its schematic

illustration.

\section{Conclusion}

We demonstrated the combination of a novel staining method with DN technique to

243 directly observe a hydrogel network at the nanoscale level. The molecular structure of

244 polymer hydrogel in bulk and on the surface was unveiled for the first time in real space. We

245 clarified the presence of dangling chains on the surface of the as-prepared gels with a length 
on the same scale as the mesh size. These results are important in understanding the surface

properties of hydrogels, including surface wetting-dewetting, sliding friction, adhesion, and

bonding. Furthermore, numerous micro-scale defects noted in these gels can induce stress

concentration of the nanoscale polymer strands. These results are essential in understanding

the origin of the brittleness of hydrogels. This method has the potential to be widely used not

only for hydrogels but also for single macromolecular level imaging of polymer materials.

\section{Experimental Methods} solution in a mold, which was made of a $2 \mathrm{~mm}$ thick silicone spacer sandwiched between two glass plates, by UV polymerization for $8 \mathrm{~h}$ in a chamber filled with inert Ar gas. Then, the PAMPS gel was removed from the mold and soaked in a PDMAAm network precursor solution 
for 1 day until reaching equilibrium. The PAMPS gel containing the PDMAAm network 270 precursor solution was sandwiched between two glass plates and irradiated with UV for its $2^{\text {nd }}$ polymerization for $6 \mathrm{~h}$ in Ar atmosphere ${ }^{[15]}$. The obtained samples were immersed in distilled water for one week to completely remove residual chemicals. All glass plates used in the molds were heated in a $500{ }^{\circ} \mathrm{C}$ oven for $1 \mathrm{~h}$ to remove any residual organics before use. The samples were coded according to the PAMPS network crosslinker ratio $\mathrm{C}_{\mathrm{MBAA}}$ (mol\%), as PAMPS$\mathrm{C}_{\text {MBAA. }}$ The thickness of these swelled PAMPS gel samples were 8.99, 5.37, and $3.36 \mathrm{~mm}$ for PAMPS-1, PAMPS-3, and PAMPS-8, respectively. PAMPS-4 micro-gels and supermacroporous PAMPS-0.5 gel were prepared by suspension polymerization and cryogelation using the protocols reported. ${ }^{[19,20]}$ The mesh size of the supermacroporous PAMPS-0.5 gel is less than that of conventional bulk PAMPS gel since the concentration of the precursor solution increases with freezing in the cryogelation. The PDMAAm network in these gels was introduced using the protocol described above.

Compression test: The compressive mechanical properties of the PAMPS gel were examined using a mechanical tester (Tensilon RTC-1310A, Orientic Co., Japan). The samples were cut into discs with $15 \mathrm{~mm}$ diameter using a cutting machine. The test was performed at a strain rate of $1 / 600 \mathrm{~s}^{-1}$. Each test was performed on five samples.

Mineral staining and transmission electron microscopy (TEM) observation: For mineral staining, the hydrogels were immersed in a staining solution of $2.5 \mathrm{M} \mathrm{FeCl}_{3}$ and $1.5 \mathrm{M} \mathrm{FeCl}_{2}$ mixture for 1 day at $25^{\circ} \mathrm{C}$. Subsequently, the gels were immersed in pure water to increase $\mathrm{pH}$ and amorphous ferric oxide nanoparticles were mineralised on the PAMPS network. TEM observations (H-7650, Hitachi, Japan) were performed to observe the polymer network morphology of the stained hydrogels. The specimens for TEM observations were prepared by freezing the hydrogels in liquid nitrogen and the water of the hydrogels was substituted with ethanol and then acrylic resin (London Resin white, medium) in the chamber of an automatic freeze substitution system (EM AFS2, Leica Microsystems, Germany). The gel thickness in 
these processes is shown in Figure S1. For all PAMPS gels with different formulations, the final thickness changes of the resin-cured specimen are within $10 \%$ relative to that of the PAMPS gels in water. Then, $100 \mathrm{~nm}$ thick resin-cured specimens were cut using an ultramicrotome knife (EM UC7i, Leica Microsystems, Germany) and then placed on a carbonsupported copper mesh grid. The electron gun has an acceleration voltage of $100 \mathrm{kV}$. Subsequently, 3D TEM and energy dispersive X-ray spectrometry were performed using a TEM tomographic system (JEM-1400 and EM-05500TGP, JEOL, Japan) and scanning electron microscope (JEM-F200, JEOL, Japan). The acceleration voltage of the electron guns was 120 and $200 \mathrm{kV}$, respectively. Image software $^{[31]}$ was used to calculate the total chain length in the TEM image of PAMPS-1.5 (Figure S3). The original TEM images were skeletonised and the total pixels were measured in the image of five samples. This total chain length is considered as the apparent length in $2 \mathrm{D}$. When a three-dimensional straight line is projected in $2 \mathrm{D}$, the $2 \mathrm{D}$ apparent length can be written by the original length multiplied by $\cos \theta$, where $\theta$ is the angle of the line with respect to the projection plane. The average value of $\cos \theta$ in the range of $0^{\circ}$ to $180^{\circ}$ is $2 / \pi$, which is approximately 0.64 .

Data analysis: The theoretical network strand contour length was calculated from the PAMPS concentration in the specimen after resin exchange. The gel composition was PAMPS1.5. The PAMPS monomer unit charged concentration at synthesis was $1 \mathrm{M}$. After equilibrium swelling, staining, and resin exchange, the PAMPS-1.5 hydrogel has a volume swelling ratio of 166 relative to its as-prepared state. Thus, the TEM sample has a PAMPS monomer unit concentration of 1/166 M. By considering the Avogadro constant of $6.02 \times 10^{23}\left(\mathrm{~mol}^{-1}\right)$ :

$$
\begin{gathered}
\text { Number of monomers per volume }=\frac{1}{166}\left(\frac{\mathrm{mol}}{\mathrm{L}}\right) \times 6.02 \times 10^{23}\left(\mathrm{~mol}^{-1}\right) \\
=3.6 \times 10^{24}\left(\text { monomer } / \mathrm{m}^{3}\right) .
\end{gathered}
$$

In this experiment, the view field has a volume of $1200 \times 1200 \times 100 \mathrm{~nm}^{3}=1.44 \times 10^{-19} \mathrm{~m}^{3}$. Thus,

$$
\text { Monomer in the view field }=3.6 \times 10^{24}\left(\frac{\text { monomer }}{m^{3}}\right) \times 1.44 \times 10^{-19}\left(\mathrm{~m}^{3}\right)
$$




$$
=518400 \text { (monomer). }
$$
theoretical network strand contour length in the fully stretched state, excluding the blob and dangling chains, is:

Total polymer length in the view field $=518400($ monomer $) \times 0.25(\mathrm{~nm} /$ monomer $)=$ $1.3 \times 10^{5}(\mathrm{~nm})$

\section{Supporting Information}

Supporting Information is available from the Wiley Online Library or from the author.

\section{Acknowledgements}

R.K., T.N. and J.P.G. designed the experiments and interpreted the results. R.K. performed the almost experiments and analysed the data. T.S. prepared the supermacroporous gel. H.J. performed STEM measurements. R.K., T.N. and J.P.G. wrote the paper. This research was financially supported by a Grant-in-Aid for Scientific Research (No. JP17H06144, JP17H06376), a Grant-in-Aid for JSPS Research Fellow (No. JP16J05057), and the Ambitious Leaders Program. J. P. Gong thanks the Institute for Chemical Reaction Design and Discovery (ICReDD) established by the World Premier International Research Initiative (WPI), MEXT, Japan. H. J. is grateful to the JSPS Kakenhi for the partial support of this research through Grant No. 19H00905. R. K. thanks Kazuki Fukao, Takahiro Matsuda, and Taiki Fukuda (Hokkaido University) for their useful comments. The authors also thank 
342 their kind help with the STEM measurements.

\section{References}

345 [1] A. S. Hoffman, Adv. Drug Deliv. Rev. 2012, 64, 18.

346 [2] O. Erol, A. Pantula, W. Liu, D. H. Gracias, Adv. Mater. Technol. 2019, 4, 1.

347 [3] R. A. Green, S. Baek, L. A. Poole-Warren, P. J. Martens, Sci. Technol. Adv. Mater. 2010, 11, DOI 10.1088/1468-6996/11/1/014107.

[4] T. Sekitani, T. Yokota, K. Kuribara, M. Kaltenbrunner, T. Fukushima, Y. Inoue, M. $10.1038 /$ ncomms 11425.

[5] M. Rubinstein, R. H. Colby, Polymer Physics, Oxford Univ. Press, Oxford, 2003.

[6] M. Shibayama, Macromol. Chem. Phys. 1998, 199, 1.

354 [7] H. Furukawa, K. Horie, Phys. Rev. E 2003, 68, 1.

355 [8] G. R. Newman, B. Jasani, E. D. Williams, Histochem. J. 1983, 15, 543.

356 [9] N. A. Ranson, P. G. Stockley, Emerg. Top. Phys. Virol. 2010, 1.

357 [10] R. Vitali, E. Montani, Polymer (Guildf). 1980, 21, 1220.

358 [11] H. Jinnai, T. Higuchi, X. Zhuge, A. Kumamoto, K. J. Batenburg, Y. Ikuhara, Acc. 
[12] R. Aso, H. Kurata, T. Namikoshi, T. Hashimoto, S. W. Kuo, F. C. Chang, H.

[13] M. Tosaka, R. Danev, K. Nagayama, Macromolecules 2005, 38, 7884.

[14] C. M. Flynn, Chem. Rev. 1984, 84, 31.

[15] J. Gong, Y. Katsuyama, T. Kurokawa, Y. Osada, Adv. Mater. 2003, 15, 1155.

[16] K. Yasuda, N. Kitamura, J. P. Gong, K. Arakaki, H. J. Kwon, S. Onodera, Y. M. Chen,

[17] J. P. Gong, Soft Matter 2010, 6, 2583.

[21] H. Jinnai, R. J. Spontak, T. Nishi, Macromolecules 2010, 43, 1675.

[22] K. Fukao, T. Nakajima, T. Nonoyama, T. Kurokawa, T. Kawai, J. P. Gong, Macromolecules 2020, 53, 1154.

[23] J. Katta, Z. Jin, E. Ingham, J. Fisher, Med. Eng. Phys. 2008, 30, 1349. 
379 [25] T. Nakajima, H. Furukawa, Y. Tanaka, T. Kurokawa, Y. Osada, J. P. Gong,

$380 \quad$ Macromolecules 2009, 42, 2184.

381 [26] M. Frauenlob, D. R. King, H. Guo, S. Ishihara, M. Tsuda, T. Kurokawa, H. Haga, S.

382 Tanaka, J. P. Gong, Macromolecules 2019, 52, 6704.

383 [27] A. A. Griffith, Masinovedenie 1995, C, 163.

384 [28] M. Marder, J. Fineberg, Phys. Today 1996, 49, 24.

385 [29] S. Seiffert, Polym. Chem. 2017, 8, 4472.

386 [30] N. Ide, T. Fukuda, Macromolecules 1999, 32, 95.

387 [31] C. A. Schneider, W. S. Rasband, K. W. Eliceiri, Nat. Methods 2012, 9, 671.

388

389

390

391

392

393

394

395

396

397 


\section{Supporting Information}

399

400

401

402

403

404

\section{Single-Macromolecular Level Imaging of a Hydrogel Structure}

Ryuji Kiyama, Takayuki Nonoyama, Tomas Sedlacik, Hiroshi Jinnai, and Jian Ping Gong*

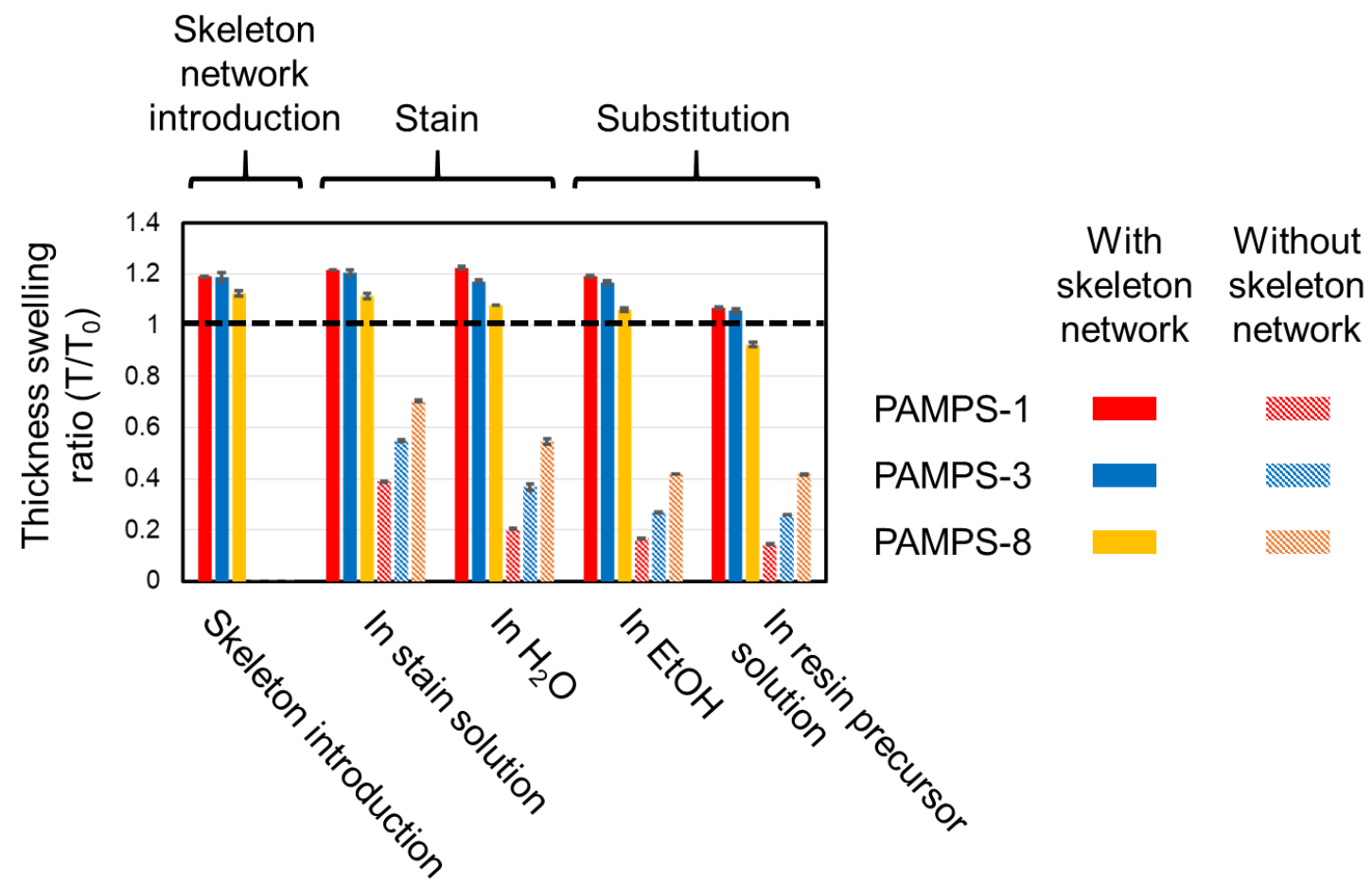

405

Figure S1. Thickness swelling ratio of the PAMPS gels with different crosslinker density during staining and substitution. $\mathrm{T}_{0}$ is the thickness of the $\mathrm{SN}$ gels swelled in water. The black dashed 408 line in the graph indicates the constant size $\left(\mathrm{T} / \mathrm{T}_{0}=1\right)$. By introducing the PDMAAm skeleton, the gel swells slightly $\left(\mathrm{T} / \mathrm{T}_{0}=1.1-1.2\right)$. The samples without a skeleton network significantly shrink $\left(\mathrm{T} / \mathrm{T}_{0}=0.1-0.4\right)$. With the skeleton network, the change in the final size of the resin-cured specimen is within $10 \%\left(\mathrm{~T} / \mathrm{T}_{0}=0.9-1.1\right)$. The error bars indicate the standard deviation for five samples. 

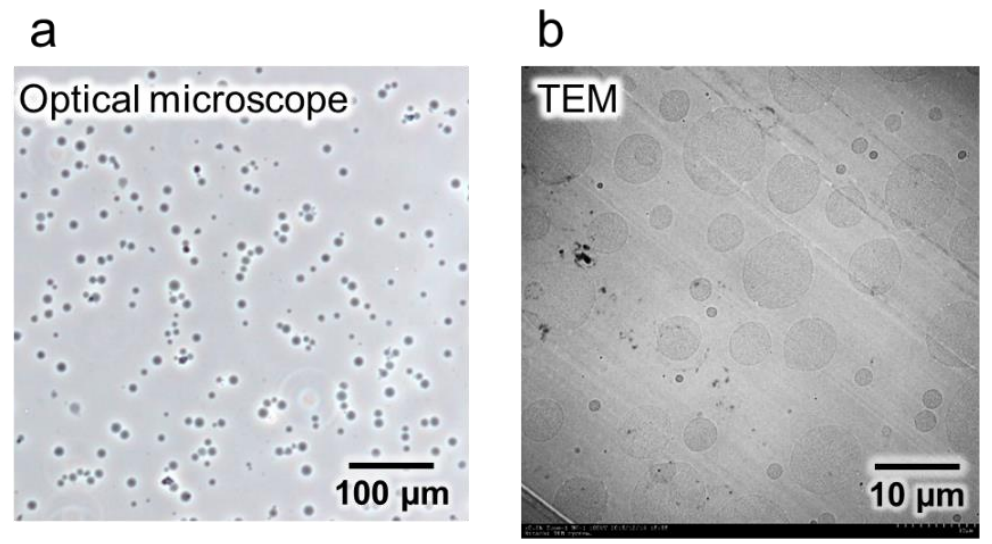

C
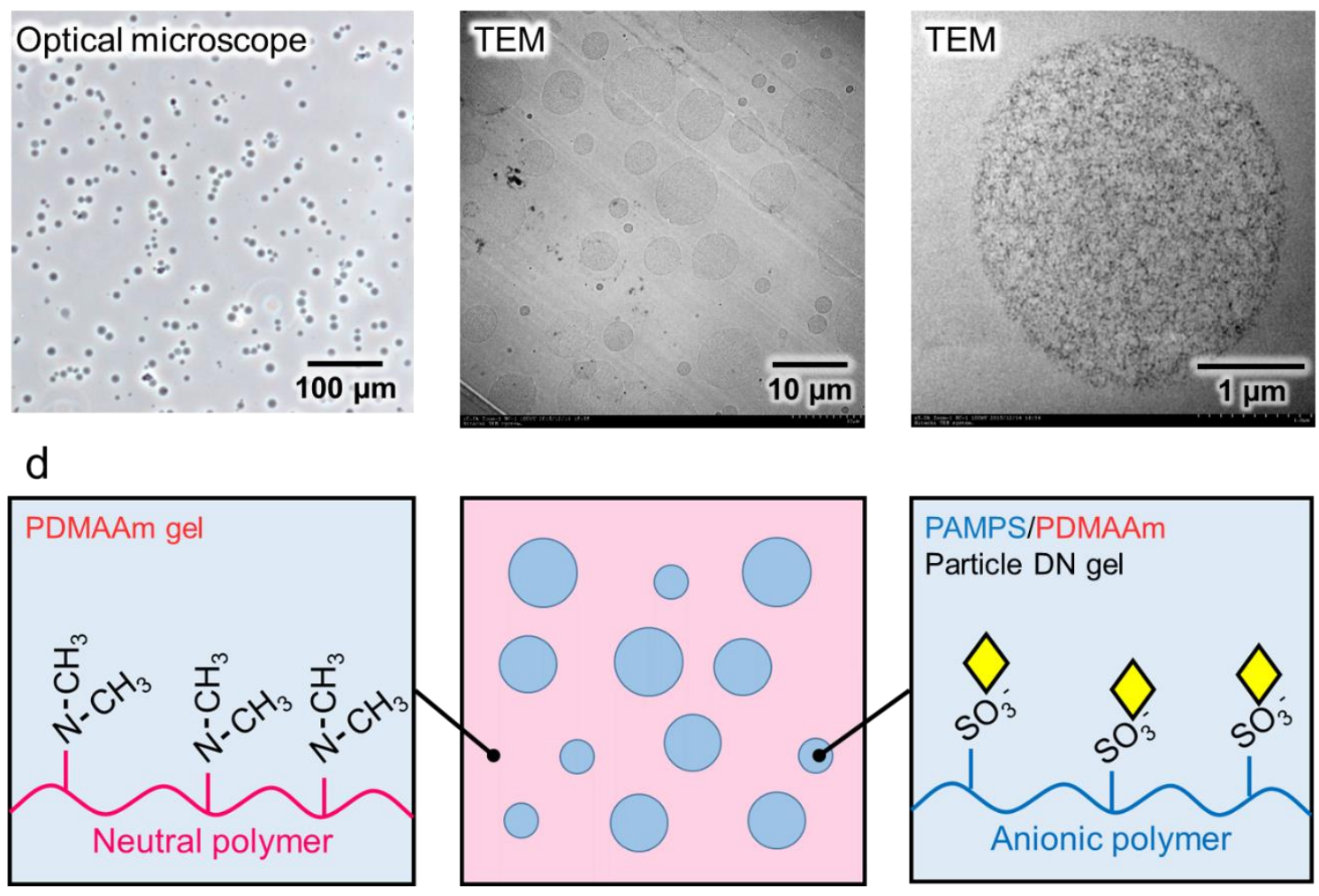

417 Figure S2. TEM image of the particle PAMPS-4 micro-gels in the bulk PDMAAm gel. (a)

418 Optical microscopy image of the pristine PAMPS micro-gel particles. (b) Low magnification 419 (×300) TEM image. $($ c) High magnification $(\times 5000)$ TEM image. $($ d $)$ Schematic illustration of 420 (b). 

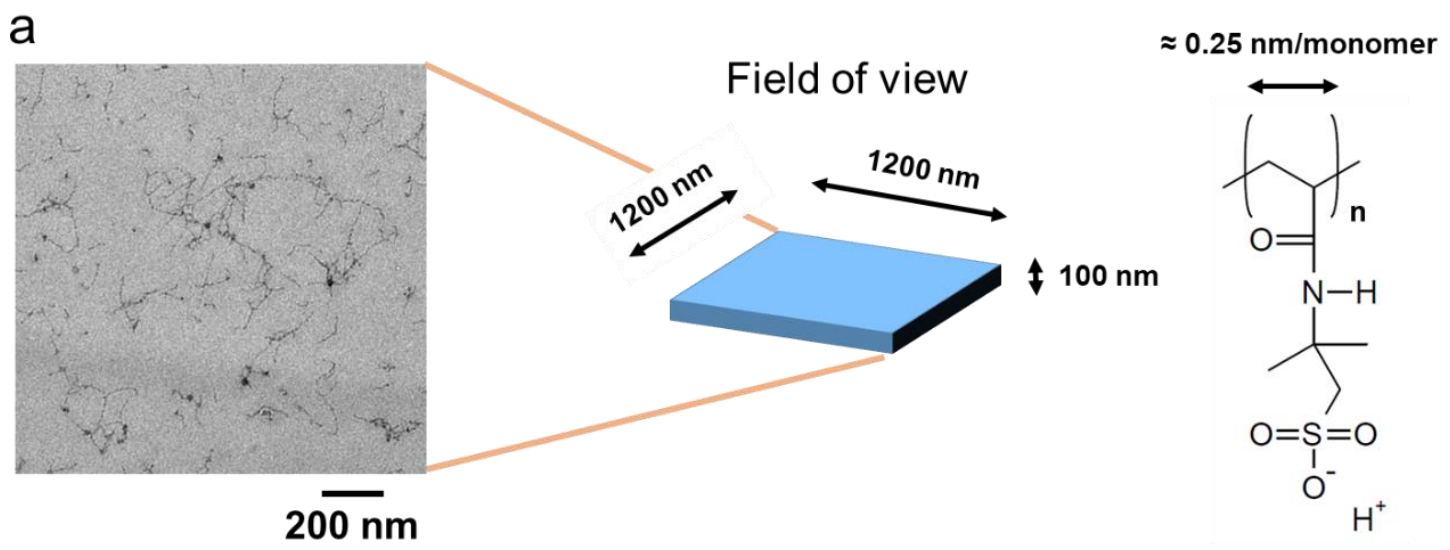

b
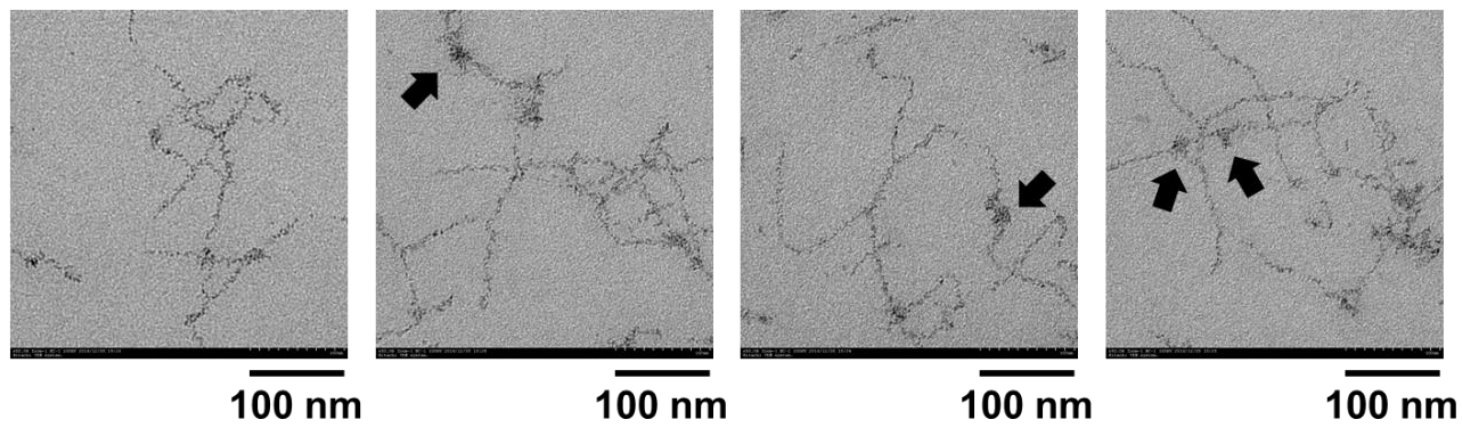

Figure S3. (a) Calculation process of the polymer length in the field of view for the PAMPS1.5 gel. The volume swelling ratio at the measured state was 166 times that of the as-prepared state for this sample. (b) High magnification TEM images of the PAMPS network at different 433 locations. The mesh structures sized in tens of nanometres were clearly observed. The black arrows indicate the apparent aggregated dangling chains in the structure. 

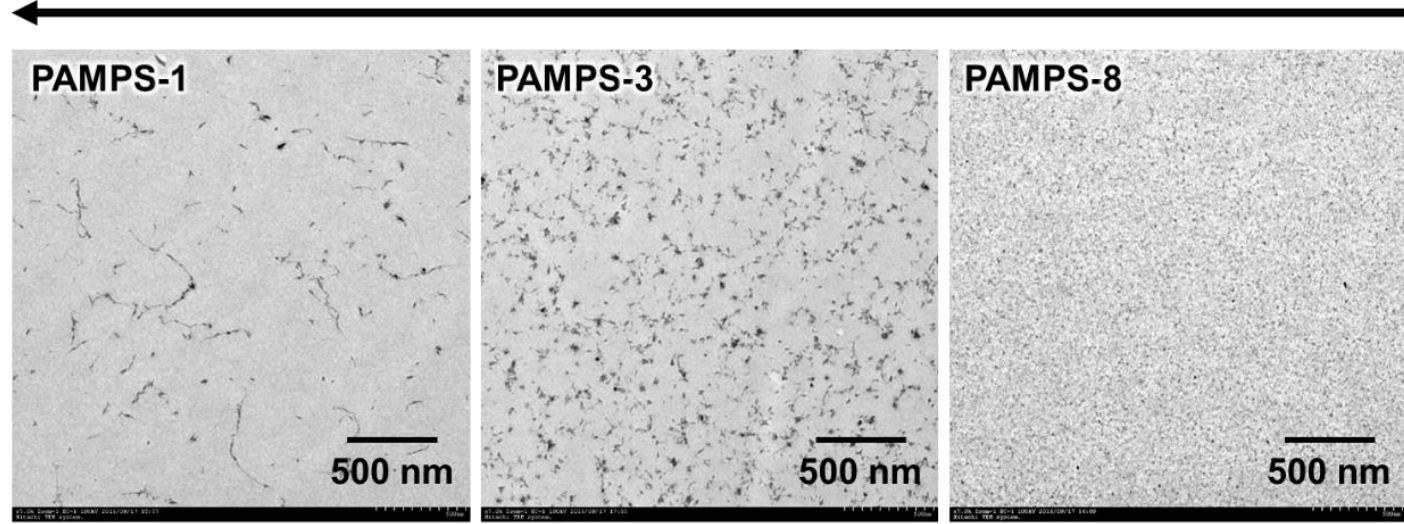

b

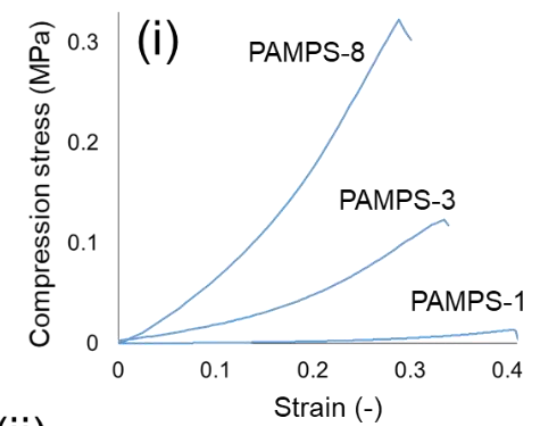

(ii)

\begin{tabular}{c|c|c} 
PAMPS & $\begin{array}{c}\text { Young's } \\
\text { Modulus (MPa) }\end{array}$ & $\begin{array}{c}\text { Monomer unit } \\
\text { concentration (M) }\end{array}$ \\
\hline 1 & $0.01 \pm 0.00$ & $0.01 \pm 0.00$ \\
3 & $0.21 \pm 0.00$ & $0.07 \pm 0.00$ \\
8 & $0.87 \pm 0.06$ & $0.24 \pm 0.00$
\end{tabular}

C Low chain density (large $\xi)$
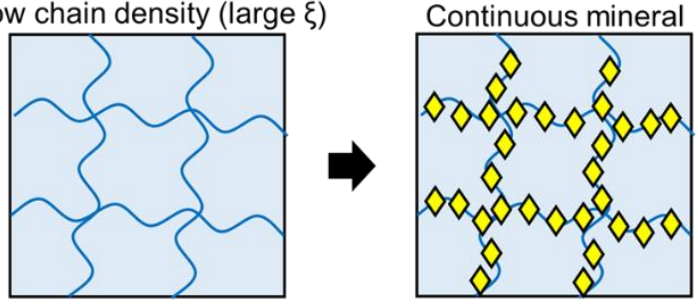

High chain density (small ६)

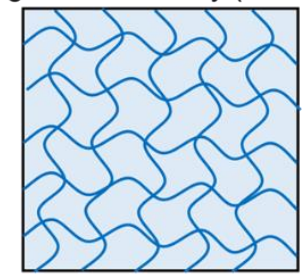

Discontinuous mineral

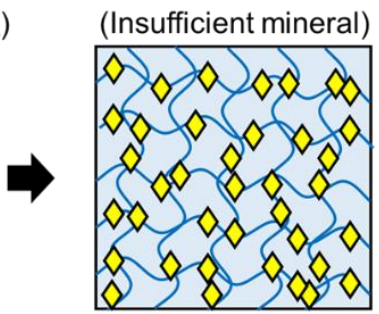

Figure S4. Effect of crosslinker ratio of the PAMPS gels. (a) TEM images. (b) (i) Stress-strain curves of the PAMPS gels in their water swollen state; (ii) Gel properties. (c) Illustrations of the network structure with low (upper) and high (lower) crosslinker density of the PAMPS gels before and after mineral staining. 


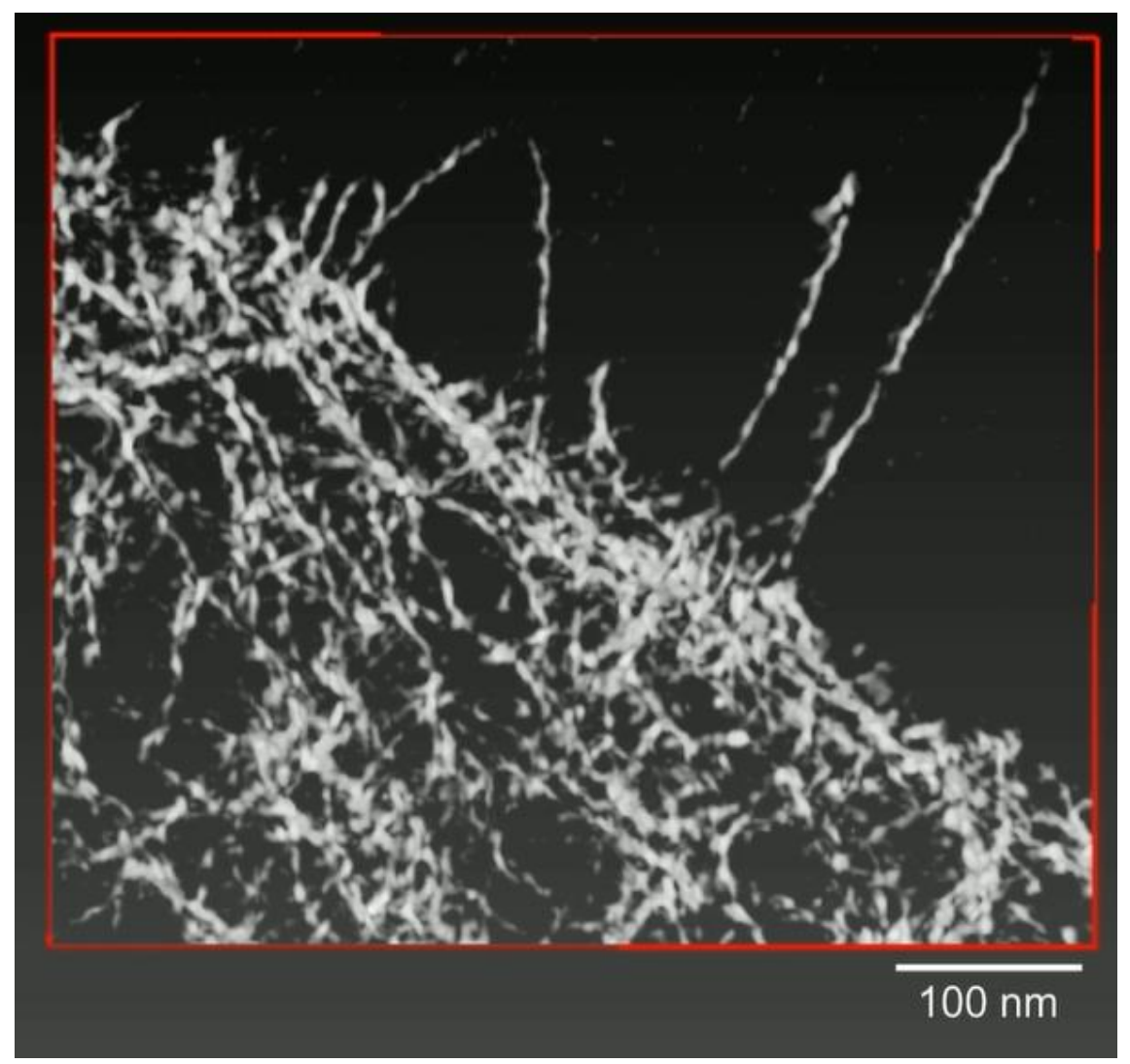

451 Supplementary Video 3D TEM movie of the supermacroporous PAMPS gel. 